\title{
Real-time intravascular photoacoustic-ultrasound imaging of lipid- laden plaque at speed of video-rate level
}

\author{
Jie Hui ${ }^{\mathrm{a}}$, Yingchun Cao ${ }^{\mathrm{b}}$, Yi Zhang ${ }^{\mathrm{a}}$, Ayeeshik Kole ${ }^{\mathrm{b}, \mathrm{c}}$, Pu Wang ${ }^{\mathrm{b}}$, Guangli Yu ${ }^{\mathrm{d}}$, Gregory Eakins ${ }^{\mathrm{e}}$, \\ Michael Sturek $^{\mathrm{b}, \mathrm{c}}$, Weibiao Chen ${ }^{\mathrm{d}, \mathrm{f}}$, Ji-Xin Cheng ${ }^{* b, g, h}$ \\ ${ }^{a}$ Department of Physics and Astronomy, Purdue University, West Lafayette, IN 47907, USA; \\ ${ }^{b}$ Weldon School of Biomedical Engineering, Purdue University, West Lafayette, IN 47907, USA; \\ ${ }^{c}$ Department of Cellular and Integrative Physiology, Indiana University School of Medicine, \\ Indianapolis, IN 46202, USA; 'Nanjing Institute of Advanced Laser Technology, Nanjing 210038, \\ China; ${ }^{\mathrm{e}}$ Jonathan Amy Facility for Chemical Instrumentation, Purdue University, West Lafayette, IN \\ 47907, USA; ${ }^{\mathrm{f}}$ Shanghai Institute of Optics and Fine Mechanics, Chinese Academy of Sciences, \\ Shanghai 201800, China; ${ }^{g}$ Department of Chemistry, Purdue University, West Lafayette, IN 47907,

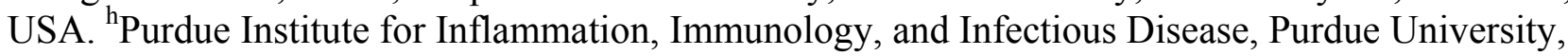 \\ West Lafayette, IN 47907, USA. \\ *Corresponding author: jcheng@purdue.edu
}

\begin{abstract}
Intravascular photoacoustic-ultrasound (IVPA-US) imaging is an emerging hybrid modality for the detection of lipidladen plaques by providing simultaneous morphological and lipid-specific chemical information of an artery wall. The clinical utility of IVPA-US technology requires real-time imaging and display at speed of video-rate level. Here, we demonstrate a compact and portable IVPA-US system capable of imaging at up to 25 frames per second in real-time display mode. This unprecedented imaging speed was achieved by concurrent innovations in excitation laser source, rotary joint assembly, $1 \mathrm{~mm}$ IVPA-US catheter, differentiated A-line strategy, and real-time image processing and display algorithms. By imaging pulsatile motion at different imaging speeds, 16 frames per second was deemed to be adequate to suppress motion artifacts from cardiac pulsation for in vivo applications. Our lateral resolution results further verified the number of A-lines used for a cross-sectional IVPA image reconstruction. The translational capability of this system for the detection of lipid-laden plaques was validated by ex vivo imaging of an atherosclerotic human coronary artery at 16 frames per second, which showed strong correlation to gold-standard histopathology.
\end{abstract}

Keywords: photoacoustic, intravascular imaging, real-time, overtone absorption, lipid, atherosclerosis, video-rate imaging

\section{INTRODUCTION}

Coronary artery disease remains the leading cause of morbidity and mortality throughout the world. Atherosclerosis, a major form of coronary artery disease, occurs in many different forms and can be distinguished by morphologic classification into different plaque types [1]. Among them, the thin-capped fibroatheroma has been widely understood to be the most "vulnerable" plaque type, as evidence shows it is the most prone to rupture and progress to thrombosis and acute coronary syndrome [1-3]. As a hybrid modality, intravascular photoacoustic-ultrasound (IVPA-US) imaging is considered a promising approach to provide more advanced and quantitative assessment of plaque lesions. Harnessing the superior penetration depth and chemical selectivity of PA imaging, IVPA channel maps the lipid deposition over the entire artery wall at $1.7 \mu \mathrm{m}$; while IVUS channel provides simultaneous and complementary morphological information. Thus far, several research groups are actively working on this technology to advance its clinical translation, which includes the identification of various tissue components [4-6], contrast mechanism [4, 7], optical excitation sources [810], IVPA-US catheter designs [10-13], and ex vivo and preclinical validations [14, 15]. However, all these works were limited by the use of slow imaging speeds as well as lack of real-time image display, which are necessary components for future in vivo applications where imaging must be at a sufficient speed to avoid motion artifacts from cardiac pulsation. Motion artifacts can lead to inaccurate spatial mapping and quantification of lipid deposition, and subsequent

Photons Plus Ultrasound: Imaging and Sensing 2017, edited by Alexander A. Oraevsky, Lihong V. Wang, Proc. of SPIE Vol. 10064, 100640T · @ 2017 SPIE · CCC code: 1605-7422/17/\$18 · doi: 10.1117/12.2251511 
misinterpretation of plaque type. Furthermore, a lack of real-time processing and display capability would prevent user feedback necessary to adjust imaging parameters and location.

Here, we overcame these limitations by demonstrating a compact and portable IVPA-US system capable of imaging in real-time, up to 25 frames per second (fps). We presented the key innovations in the system to enable the video-rate level imaging speed, which include excitation laser source, rotary joint assembly, IVPA-US catheter probe, differentiated Aline strategy, and real-time image processing and display algorithms. We characterized system spatial resolution to support the use of A-line numbers in a cross-sectional IVPA image and imaged phantom with pulsatile motion to find the adequate imaging speed for in vivo applications. Using this system, we further imaged a diseased human coronary artery ex vivo with comparison to gold standard histopathology, demonstrating its translational capability.

\section{METHODS}

\section{$2.11 .7 \mu \mathrm{m} 2 \mathrm{kHz}$ MOPA-pumped OPO}

In order to achieve high-speed imaging, we used a custom-built master oscillator power amplifier (MOPA)-pumped optical parametric oscillator (OPO) with a $2 \mathrm{kHz}$ optical excitation at $1.7 \mu \mathrm{m}$. The detailed optical layout was shown in Fig. 1(a). Figs. 1(b)-(e) showed the key performance parameters of the laser output. The wavelength was measured to be $1725 \mathrm{~nm}$, which coincides with the first overtone transition frequency of C-H bonds and is optimal for lipid excitation. The output power was tunable in a range of $2.1 \mathrm{~W}$, sufficient for lipid excitation. The pulse duration was $11.46 \mathrm{~ns}$. The pulse-to pulse energy variation was at 5.6\%, making it not necessary to normalize the pulse energy for each A-line. Collectively, this laser source was optimal for high-speed IVPA imaging of lipids.
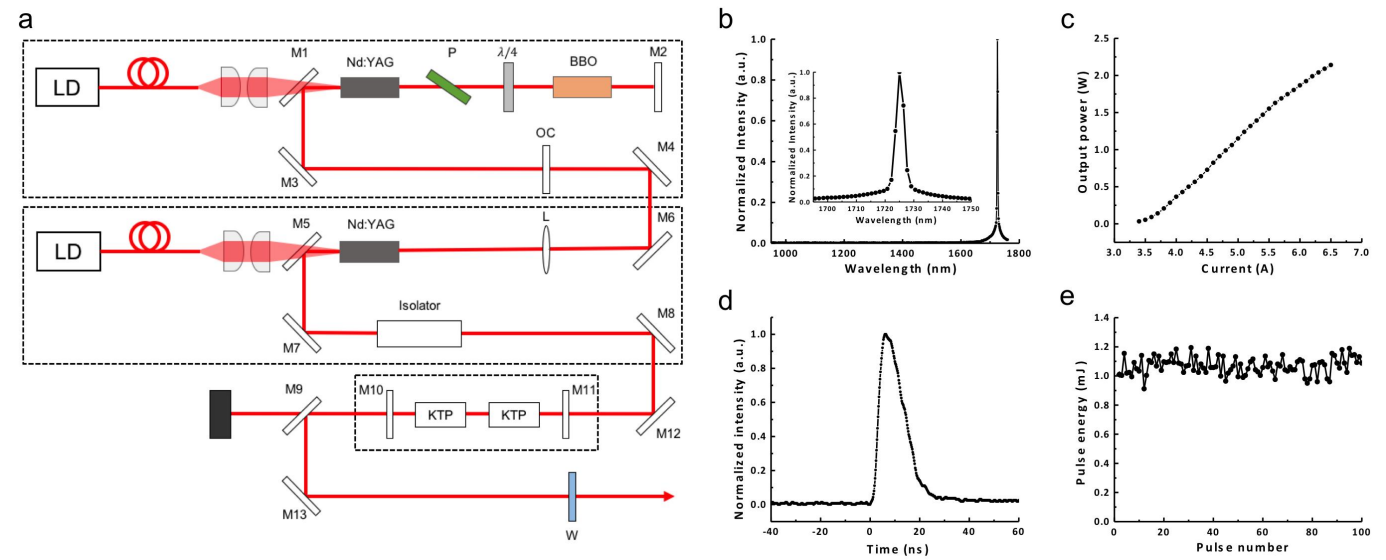

Figure 1. 1.7 $\mu \mathrm{m} 2 \mathrm{kHz}$ MOPA-pumped OPO for high-speed optical excitation. (a) Schematic of MOPA-pumped OPO. Dashed boxes labeled by I, II, and III highlight the master oscillator, optical power amplifier, and OPO, respectively. LD, laser diode; M1 and M5, fold mirror; M2, M10, and M11, flat mirror; M3, M4, M6, M7, M8, M12, and M13, reflective mirror; M9, dichroic mirror; P, polarizer; BBO, beta barium borate Pockels cell; OC, output coupler; L, lens; KTP, potassium titanyl phosphate; W, output window. (b) Output wavelength. (c) Output power with control current. (d) Output pulse duration. (e) Output pulse-to-pulse variation. 


\subsection{IVPA-US catheter and fiber-optic rotary joint assembly}
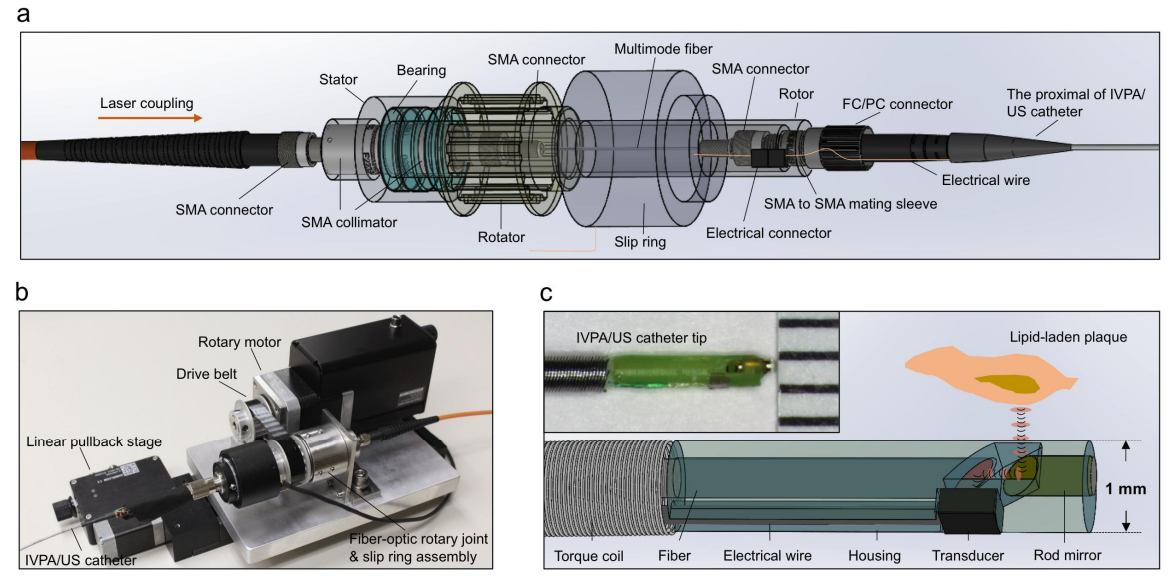

Figure 2: Fiber-optic rotary joint assembly and high-sensitivity catheter. (a) Schematic of fiber-optic rotary joint for highpulse-energy high-repetition-rate laser delivery. (b) Picture of the overall fiber-optics rotary joint assembly. (c) $1 \mathrm{~mm}$ diameter collinear IVPA-US catheter design. The inset shows the picture of the assembled catheter. Ruler scale: $1 \mathrm{~mm}$.

We designed and assembled a fiber-optic rotary joint assembly to support the high-speed imaging (Figs. 2(a) and 2(b)). In this design, the use of two adjacent collimators and a mating sleeve enabled an overall coupling efficiency of $60 \%$ from the initial optical input to the final output at the catheter tip and a coupling efficiency variation of $5.4 \%$ caused by mechanical rotation. Thus, the artery wall was uniformly excited in laser pulse energy at every angular position. Rotation speed of 30 revolutions per second was achieved through the fiber-optic rotary joint. The IVPA-US catheter implemented in the system had a collinear design (detailed design can be found in [11]) and a clinically relevant diameter of $1 \mathrm{~mm}$ (Fig. 2(c)). The reduction in catheter diameter was achieved by aligning a miniaturized single element transducer $\left(0.5^{*} 0.6^{*} 0.2 \mathrm{~mm}^{3}\right)$, a multimode fiber with core diameter of $365 \mu \mathrm{m}$, and a reflection rod mirror with diameter of $365 \mu \mathrm{m}$ in a 3-D printed housing. The inset in Fig. 2(c) showed the actual image of an assembled IVPA-US catheter tip.

\subsection{Real-time video-rate IVPA-US imaging system}
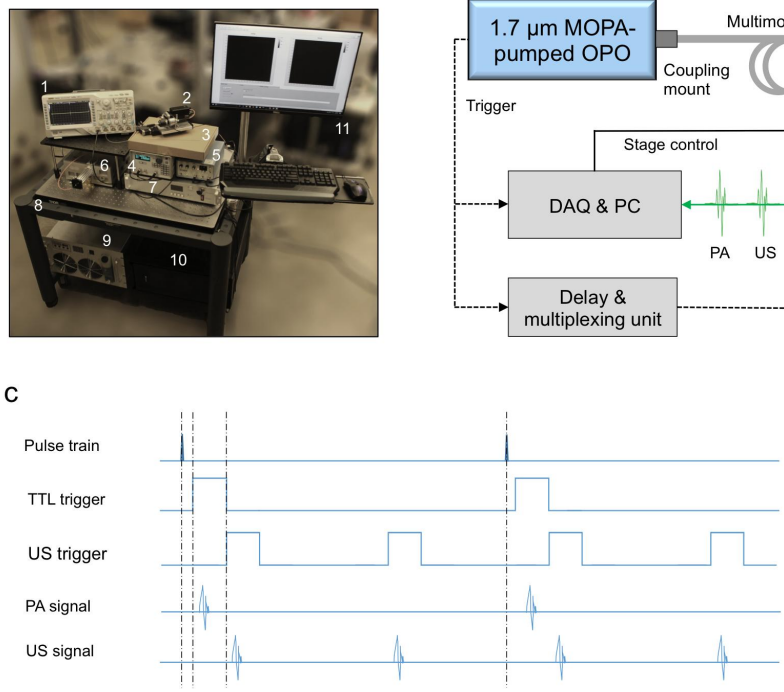

b
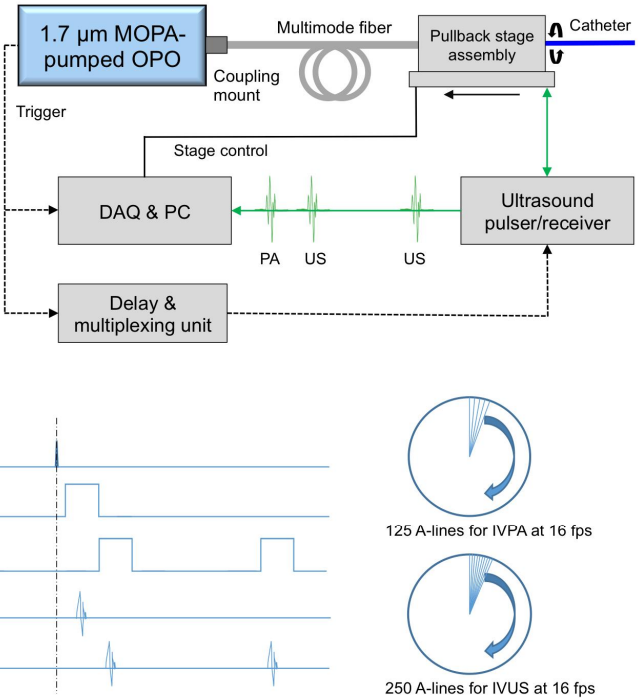

Figure 3: Real-time video-rate IVPA-US imaging system. (a) Picture of the compact and portable IVPA-US system. Major components: 1, oscilloscope; 2, fiber-optic rotary joint assembly; 3, IVPA-US catheter; 4, delay generator; 5, ultrasound pulser/receiver; 6, MOPA-pumped OPO; 7, laser controller; 8, mobile cart; 9, laser chiller; 10, PC and data acquisition card; 11, image display monitor. (b) IVPA-US imaging system layout. Dashed black line: trigger signal; solid black line: stage control cable; solid green line: radiofrequency signal. (c) Differentiated A-line strategy for IVPA and IVUS imaging and its timing diagram. 
We designed and developed the compact and portable IVPA-US imaging system as shown in Fig. 3(a). The detailed connections and controls of these components were shown in Fig. 3(b). The MOPA-pumped OPO was implemented into the system for high-speed optical excitation. Its output pulses were coupled to a multimode fiber through a coupling mount, then a fiber-optic rotary joint, and lastly the IVPA-US catheter tip. Initial ultrasound pulses generated from an ultrasound pulser were sent to the catheter tip through a slip ring. The delays between the ultrasound and optical pulses were precisely controlled by a delay generator. The B-scan and linear pullback of the catheter was conducted with the fiber-optic rotary joint assembly and rotation and pullback rate controlled by a computer. The sequentially generated PA and US signals were detected by the IVPA-US catheter, transmitted by a slip ring, amplified by the ultrasound receiver, digitized and recorded by a data acquisition card, and further processed and displayed in real-time. Meanwhile, a differentiated A-line strategy was designed to maximize IVPA-US imaging functionality at high imaging speeds (see the timing diagram in Fig. 3(c). In this design, trigger signal for US was delayed and frequency-doubled through multiplexing by the delay generator. As a result, the digitized signal in one acquisition cycle contained one PA signal segment and two US signal segments. Thus, the number of A-lines in a cross-sectional IVUS image was two times of that in IVPA image. Specifically, at $16 \mathrm{fps}, 125$ A-lines were used to reconstruct a cross-sectional IVPA image; 250 Alines was used to reconstruct a IVUS image, comparable to current commercial systems. Furthermore, LabView-based algorithms were developed for IVPA-US image processing and display in parallel and in real-time.

\section{RESULTS}

\subsection{IVPA lateral resolution characterization}

In order to verify the A-line number used in a cross-sectional IVPA image, we characterized IVPA lateral resolution by imaging of a single $30 \mu \mathrm{m}$ diameter carbon fiber submerged in heavy water. The cross-sectional IVPA image at $16 \mathrm{fps}$ was shown in Fig. 4(a) and its lateral resolution of $305 \mu \mathrm{m}$ was estimated by the full-width-at-half-maximum of Gaussian fitted raw data points in the lateral direction at an axial position of $2.38 \mathrm{~mm}$ (Fig. 4(b)). At this axial position, there were 2.5 A-lines sampled in the lateral resolution of $305 \mu \mathrm{m}$, which indicates that signals in IVPA image was adequately sampled in the lateral direction based on the Nyquist sampling theorem $(2.5$ was calculated by $\mathrm{L} /(2 * \mathrm{pi}) \mathrm{R} / \mathrm{N})$, where $\mathrm{L}$ is the later resolution, $\mathrm{N}$ is the number of A-lines per cross-sectional image, and $\mathrm{R}$ is the axial position). Using the same methodology, there were constantly $\sim 2.5$ A-lines sampled in the lateral resolution at every axial position for 16 fps IVPA imaging (calculated based on the data in Fig. 4(c)). Thus, the use of 125 A-lines should provide adequate image quality for IVPA imaging. Meanwhile, we characterized the lateral resolutions at different axial positions at other imaging speeds (Fig. 4(c)). Notably, when the axial position was increased, the lateral resolution decreased, varying from 150 to $600 \mu \mathrm{m}$. However, there was no significant difference in lateral resolution at each axial position at different imaging speeds.
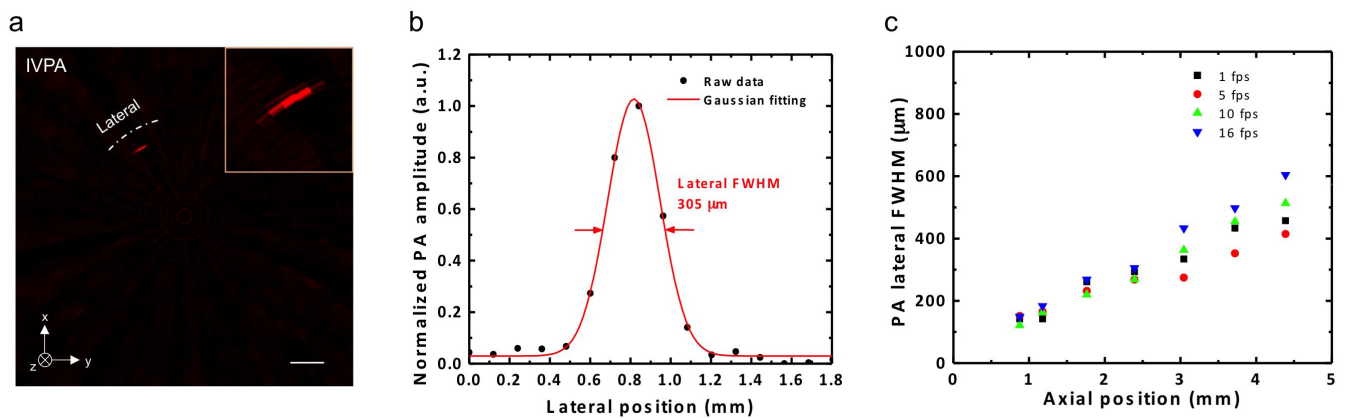

Figure 4: IVPA lateral resolution characterization. (a) Cross-sectional IVPA image of a single carbon fiber with diameter of $30 \mu \mathrm{m}$. Inset shows the zoom-in IVPA image. Scale bar: $1 \mathrm{~mm}$. (b) Lateral plot of the carbon fiber in IVPA image at an axial position of $2.38 \mathrm{~mm}$. The lateral resolution of $305 \mu \mathrm{m}$ is estimated by the full with at half maximum (FWHM) of Gaussian fitted curve. (c) IVPA lateral resolution at different axial position at imaging speeds of 1, 5, 10, and 16 fps. 


\subsection{IVPA-US imaging of phantom with pulsatile motion at different imaging speeds}

To find the optimal speed for imaging arteries with cardiac motion in in vivo settings, a phantom (a segment of heat shrink tube) with $1.2 \mathrm{~Hz}$ pulsatile motion mimicking human heartbeat was imaged at different speeds: 1, 5, 10, 16, 20, and 25 fps. Merged IVPA-US images of the dynamic motion captured at different speeds were shown in Video1. We found that the imaging speed did not affect detection sensitivity and IVPA-US co-registration. IVPA-US imaging at speeds of 1 and $5 \mathrm{fps}$ was not able to accurately capture the $1.2 \mathrm{~Hz}$ pulsatile motion. The speed of $10 \mathrm{fps}$ was acceptable, but its slow refresh rate was still noticeable. However, there was no observable difference between the speeds of 16, 20, $25 \mathrm{fps}$ in capturing the $1.2 \mathrm{~Hz}$ pulsatile motion. Thus, IVPA-US images at low imaging speeds were subject to distortion from motion artifacts. As contrasting examples, four consecutive IVPA-US images at 1 fps (Fig. 5(a)) and 16 fps (Fig. 5(b)) were used to demonstrate the distortion. First, at $1 \mathrm{fps}$, there was obvious A-line signal mismatch at 6 o'clock, where the first and final A-lines in a cross-sectional image were located (Frames \#2 and \#4 in Fig. 5(a)). However, at 16 fps, the mismatch became negligible (Fig. 5(b)). Furthermore, the overall shape of shrink tube at $1 \mathrm{fps}$ was distorted (for all the frames in Fig. 5(a)); while the distortion was successfully suppressed at $16 \mathrm{fps}$ and the images reflected the real cross-sections of the tube (Fig. 5(b)). Lastly, at 16 fps, the dynamic change on tube shape from Frame \#1 to \#4 in Fig. 5(b) was continuous; but, at $1 \mathrm{fps}$, such continuity was not shown (Fig. 5(a)). Thus, a 16 fps imaging speed should be sufficient to avoid motion artifacts induced by cardiac pulsation for in vivo IVPA-US imaging.
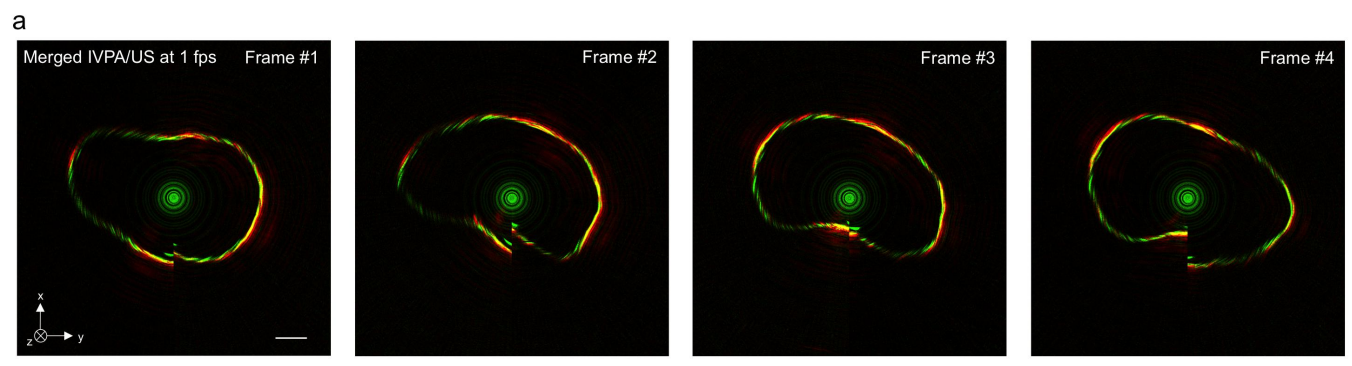

b
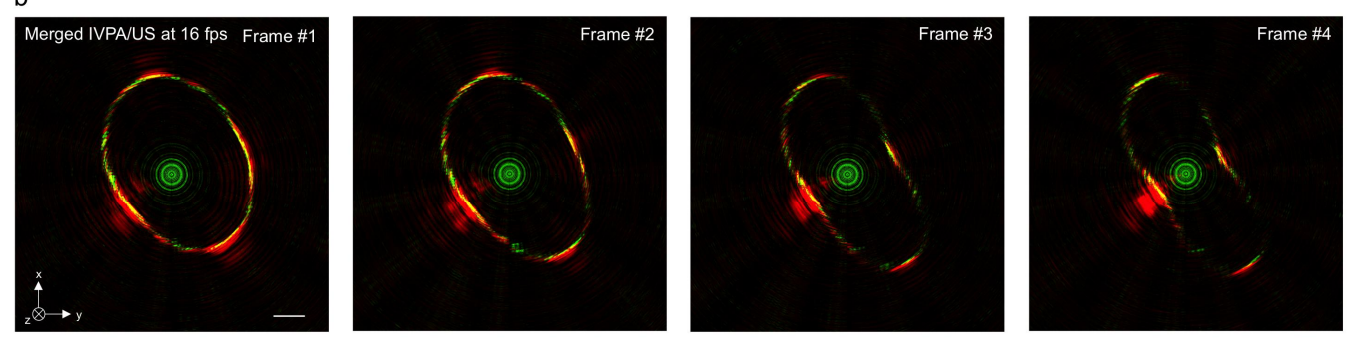

Figure 5: IVPA-US imaging of heat shrink tube with heartbeat mimicking pulsatile motion at different speeds. (a) four consecutive IVPA-US image frames at 1 fps highlighting motion induced image distortion. (b) four consecutive IVPA-US image frames at $16 \mathrm{fps}$ showing the capability to monitor the pulsatile motion. The depth field of view is $5.09 \mathrm{~mm}$. Scale bar: $1 \mathrm{~mm}$. The red and green colors represented IVPA and IVUS signals, respectively. Videol showed the complete comparison of IVPA-US imaging results at speed of 1, 5, 10, 16, 20, and $25 \mathrm{fps}$. http://dx.doi.org/10.1117/12.2251511

\subsection{IVPA-US imaging of human coronary atherosclerosis with comparison to histopathology}

To validate the translational capability of imaging system for the detection of lipid-laden plaques, we further imaged a diseased human coronary artery ex vivo. The coronary artery sample was dissected from a human heart, collected from a 44-year old male who died of atherosclerotic hypertensive cardiovascular disease (Fig. 6(a)). Using the system, a positive region of interest was identified based on IVPA and merged images in Figs. 6(b) and (c), where there were two sites of lipid deposition at the 2 and 8 o'clock positions, as recognized by strong signals in the IVPA channel. Notably, for the lipid-rich core at the 2 o'clock position, it further correlated with luminal encroachment observed in the IVUS channel. The co-registered IVPA-US image (Fig. 6(c)) suggested that this lipid-rich core was beneath a fibrous cap of the plaque surface shown by signal in the IVUS channel. These imaging results were further confirmed by gold-standard histopathology at the region of interest (Fig. 6(h)), where the two sites of lipid deposition were identified by the cholesterol clefts, loss of matrix, and macrophage infiltration into the lipid pool with an overlying fibrous cap $(200 \mu \mathrm{m}$ thickness). 

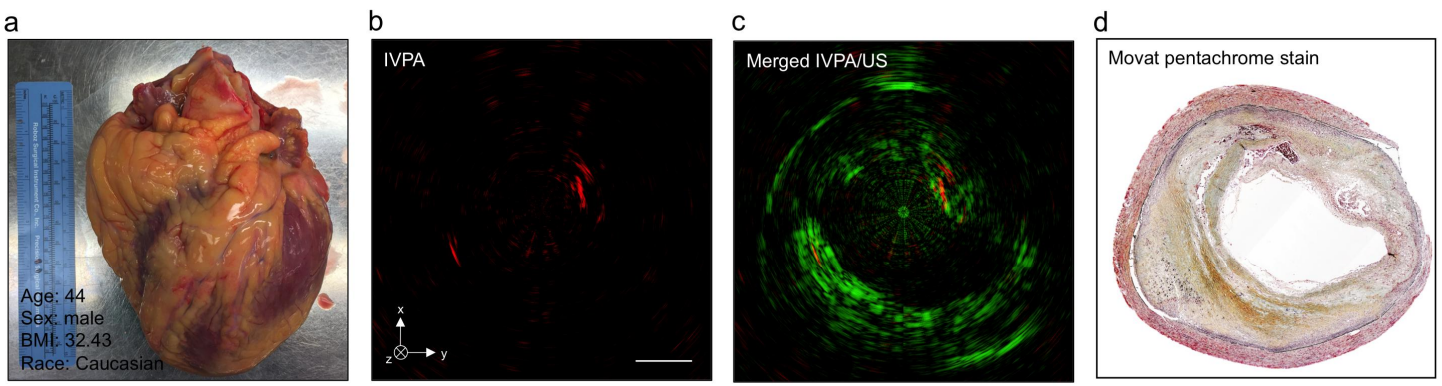

Figure 6: IVPA-US imaging of lipid-laden plaque at $16 \mathrm{fps}$ in a diseased human coronary artery. (a) Picture of human heart sample with sample information. Cross-sectional (b) IVPA and (c) merged IVPA-US images of human coronary artery at the region of interest. (d) Gold-standard histopathology stained with Movat's pentachrome at the region of interest. Scale bar: $1 \mathrm{~mm}$.

\section{CONCLUSIONS}

In summary, we have demonstrated a real-time IVPA-US system capable of imaging up to $25 \mathrm{fps}$. This speed is comparable to the current commercial IVUS and NIRS/IVUS systems. Although a video-rate speed at $30 \mathrm{fps}$ is preferred for in vivo applications, achieving so would potentially be cost-prohibitive during laser sourcing and development. By imaging phantom with pulsatile motion at different imaging speeds, our results further suggested that 16 fps was adequate to suppress motion artifacts from cardiac pulsation for future in vivo applications. Using this system, we imaged and identified a high-risk plaque, an advanced fibroatheroma, in a human coronary artery ex vivo. Collectively, this work offered significant advances towards the clinical translation of IVPA-US imaging technology.

\section{ACKNOWLEDGMENTS}

The authors thank Randy Replogle at Jonathan Amy Facility for part machining. Y.C. acknowledges AHA Postdoctoral Fellowship for financial support. A.K. acknowledges IUPUI Graduate Student Imaging Research Fellowship for financial support. This work was supported by R01HL125385 to J.-X. Cheng and M. Sturek. J.-X.C. and P.W. have a financial interest in Vibronix Inc., which does not support this work. J.H., Y.C., Y.Z., and A.K. contributed equally to this work.

\section{REFERENCES}

[1] K. Yahagi, F. D. Kolodgie, F. Otsuka et al., "Pathophysiology of native coronary, vein graft, and in-stent atherosclerosis," Nat Rev Cardiol, 13(2), 79-98 (2016).

[2] J. Narula, M. Nakano, R. Virmani et al., "Histopathologic characteristics of atherosclerotic coronary disease and implications of the findings for the invasive and noninvasive detection of vulnerable plaques," J Am Coll Cardiol, 61(10), 1041-51 (2013).

[3] A. V. Finn, M. Nakano, J. Narula et al., "Concept of vulnerable/unstable plaque," Arterioscler Thromb Vasc Biol, 30(7), 1282-92 (2010).

[4] H. W. Wang, N. Chai, P. Wang et al., "Label-free bond-selective imaging by listening to vibrationally excited molecules," Phys Rev Lett, 106(23), 238106 (2011).

[5] T. J. Allen, A. Hall, A. P. Dhillon et al., "Spectroscopic photoacoustic imaging of lipid-rich plaques in the human aorta in the 740 to $1400 \mathrm{~nm}$ wavelength range," J Biomed Opt, 17(6), 061209 (2012).

[6] B. Wang, J. L. Su, J. Amirian et al., "Detection of lipid in atherosclerotic vessels using ultrasound-guided spectroscopic intravascular photoacoustic imaging," Opt Express, 18(5), 4889-97 (2010).

[7] J. Hui, R. Li, E. H. Phillips et al., "Bond-selective photoacoustic imaging by converting molecular vibration into acoustic waves," Photoacoustics, 4(1), 11-21 (2016). 
[8] P. Wang, T. Ma, M. N. Slipchenko et al., "High-speed intravascular photoacoustic imaging of lipid-laden atherosclerotic plaque enabled by a 2-kHz barium nitrite raman laser," Sci Rep, 4, 6889 (2014).

[9] J. Hui, Q. Yu, T. Ma et al., "High-speed intravascular photoacoustic imaging at 1.7 mum with a KTP-based OPO," Biomed Opt Express, 6(11), 4557-66 (2015).

[10] Y. Li, X. Gong, C. Liu et al., "High-speed intravascular spectroscopic photoacoustic imaging at 1000 A-lines per second with a 0.9-mm diameter catheter," J Biomed Opt, 20(6), 065006 (2015).

[11] Y. Cao, J. Hui, A. Kole et al., "High-sensitivity intravascular photoacoustic imaging of lipid-laden plaque with a collinear catheter design," Sci Rep, 6, 25236 (2016).

[12] A. B. Karpiouk, B. Wang, and S. Y. Emelianov, "Development of a catheter for combined intravascular ultrasound and photoacoustic imaging," Rev Sci Instrum, 81(1), 014901 (2010).

[13] X. Bai, X. Gong, W. Hau et al., "Intravascular optical-resolution photoacoustic tomography with a $1.1 \mathrm{~mm}$ diameter catheter," PLoS One, 9(3), e92463 (2014).

[14] K. Jansen, A. F. van der Steen, H. M. van Beusekom et al., "Intravascular photoacoustic imaging of human coronary atherosclerosis," Opt Lett, 36(5), 597-9 (2011).

[15] B. Wang, A. Karpiouk, D. Yeager et al., "In vivo intravascular ultrasound-guided photoacoustic imaging of lipid in plaques using an animal model of atherosclerosis," Ultrasound Med Biol, 38(12), 2098-103 (2012). 